\title{
Bilan de la recherche scientifique en communication au Quebec - Photographie sur le vif
}

Anne-Tamara Lorre et Thérèse Paquet-Sévigny

\section{OpenEdition}

\section{Journals}

Édition électronique

URL : http://journals.openedition.org/communicationorganisation/3031

DOI : 10.4000/communicationorganisation.3031

ISSN : 1775-3546

Éditeur

Presses universitaires de Bordeaux

\section{Édition imprimée}

Date de publication : 1 novembre 1996

ISSN : 1168-5549

\section{Référence électronique}

Anne-Tamara Lorre et Thérèse Paquet-Sévigny, « Bilan de la recherche scientifique en communication au Quebec - Photographie sur le vif », Communication et organisation [En ligne], 10 | 1996, mis en ligne le 01 avril 2012, consulté le 19 avril 2019. URL : http://journals.openedition.org/ communicationorganisation/3031; DOI : 10.4000/communicationorganisation.3031

Ce document a été généré automatiquement le 19 avril 2019

(c) Presses universitaires de Bordeaux 


\title{
Bilan de la recherche scientifique en communication au Quebec - Photographie sur le vif
}

\author{
Anne-Tamara Lorre et Thérèse Paquet-Sévigny
}

\section{NOTE DE L'ÉDITEUR}

Cet article est issu d'une recherche présentée dans le cadre du séminaire

«Communication, journalisme et médias : formation et recherche » réalisé conjointement par l'Institut français de presse de l'université Panthéon - Assas Paris II, le département d'information et de communication de l'université Laval et la chaire UNESCO-BELL ; il figure dans les actes du séminaire sous le titre « Les pratiques professionnelles en communication dans la recherche scientifique au Québec ».

1 Prendre une photographie sur le vif de la recherche en communication au Québec et faire le focus sur la recherche qui étudie les pratiques professionnelles en communication, tel est l'objet de cet article qui fait suite à une étude menée au Québec à l'automne 1996.

2 Hormis le portrait dressé par Liora Salter pour le Canada au début des années 80 - un tableau riche et explicite des courants théoriques de recherche en communication - nous n'avons pas de relevé général et récent de la recherche en communication au Québec. Nous avons voulu produire cette image d'ensemble, pour souligner de ce portrait les traits saillants, faire ressortir les tendances lourdes et les segments plus fragiles, et dégager les faits porteurs d'avenir. Une fois ce portrait achevé, nous voulions évaluer la place de la recherche sur les pratiques professionnelles en communication, son importance par rapport aux autres axes de recherche, son influence éventuelle sur ces axes ${ }^{1}$ et l'impact possible sur les pratiques.

3 Inutile de dire que nous n'avons fait que lever le voile sur cette large problématique, et qu'il faudra initier d'autres cycles de réflexion à partir de cet état des lieux de la recherche. 
Une question double orienta la démarche : quelles sont les problématiques de recherche en communication négligées au Québec, en fonction desquelles nous devrions considérer des priorités de recherche à court terme, en particulier dans le domaine des pratiques professionnelles en communication?

5 Très vite, des tendances lourdes se sont dégagées des 174 projets inventoriés : tendance à la recherche sur les nouvelles technologies de l'information et de la communication (NTIC), tendance à la diminution des subventions par les organismes traditionnels, et conséquemment au regroupement des chercheurs au sein de centres de recherche multisectoriels, multidisciplinaires, interuniversitaires et internationaux, financés par de nouveaux partenaires privés. Ces nouveaux partenariats privilégient la recherche et développement, et la recherche appliquée au détriment de la recherche fondamentale, de la recherche empirique et des études de cas. Les organismes de financement eux-mêmes se regroupent dans le cadre de programmes d'action conjointe. Par ailleurs, les problématiques de recherche sur les pratiques professionnelles en communication, soit les métiers du journalisme, des relations publiques et de la publicité, sont très marginales et les publications dans ce champ de recherche sont rares. Comme il n'y a ni courant de recherche de portée internationale, ni revue faisant l'unanimité dans le domaine de la recherche en communication au Québec, la nécessité d'une démarche nationale, continentale ou internationale plus intégrée se fait sentir.

\section{Définition des concepts clés}

6 Avant d'exposer la méthodologie suivie et de détailler les résultats de cette étude, il est indispensable, dans un champ aussi mouvant et controversé que celui de la discipline " communication », d'expliciter les concepts clés sur lesquels s'appuie cette étude.

7 Au Québec comme ailleurs, la communication vue comme discipline ou au contraire comme champ disciplinaire occupe une large place dans les débats et les écrits universitaires. Certains pensent que la communication n'est pas à proprement parler une discipline, mais plutôt un champ de recherche et de pratique aux confins de nombreuses disciplines en sciences humaines comme la sociologie, la psychologie, les sciences politiques, les études littéraires, etc. D'autres soutiennent que cette jeune discipline est en voie de fédération, qu'elle est ouverte et que c'est davantage un signe de vitalité qu'elle n'aspire pas à s'enfermer dans une communicologie. Quoi qu'il en soit, il est indispensable de préciser les critères qui nous ont permis de sélectionner les chercheurs et les projets considérés dans le cadre de cette étude.

8 Les travaux de recherche des chercheurs travaillant sur le droit des communications, ou sur la gestion des arts ont été considérés; ainsi que les groupes de recherche de disciplines connexes, travaillant sur des questions reliées à l'information et aux communications, fréquemment dans le cadre de projets multidisciplinaires impliquant des chercheurs en communication, ont également retenu notre attention.

9 Il aurait été inadéquat de confiner notre étude aux seuls départements universitaires d'étude en communication puisqu'une proportion importante de la recherche en communication s'effectue à l'extérieur des départements de communication et que, d'autre part, une proportion grandissante de la recherche s'effectue en partenariat avec des institutions à l'extérieur du cadre universitaire ${ }^{2}$. C'est pourquoi le projet de recherche, et non pas le chercheur, est l'imité de base sur laquelle se fonde cette étude. 
10 Deux critères, exclusifs ou combinés, pouvaient présider à la sélection des projets qui allaient être retenus : l'objet de recherche ou la perspective du chercheur ${ }^{3}$. Le parti pris a été celui de la perspective : une recherche sur l'économie du cinéma n'est pas considérée comme une recherche en communication dans cette étude, à moins qu'elle ne s'intéresse, par exemple, aux stratégies de communication des distributeurs de films...

11 Ce parti pris favorise une conception de la communication comme discipline, tandis qu'une sélection effectuée à partir de l'objet de recherche aurait contribué à accentuer le patchwork du champ de la communication, à l'intersection des sciences humaines, économiques, politiques et sociales, et des arts et lettres.

12 La définition de ce qui devait être qualifié et reconnu comme étant de la recherche ne fut pas plus aisée. Des zones frontières de plus en plus étroites séparent la recherche de l'intervention, du conseil et du développement. La recherche qui se fait à la frontière de ces champs d'expertise, telle que la recherche-action, la recherche-évaluation, ou la recherche et développement est en croissance. Alors comment distinguer avec certitude ce qui relève de la recherche de ce qui relève exclusivement de l'intervention, du conseil ou du développement?

13 Cette fois encore, deux critères de sélection peuvent être envisagés: la nature de l'expertise développée et l'occurrence de publication. De fait, les activités de conseil, d'évaluation ou de développement donnent rarement lieu à des publications savantes. Cependant, par acquit de conscience, nous avons combiné ces deux critères. Ainsi par exemple, au niveau micro, plusieurs recherche et développement sur les multimédias se réduisent à une dimension informatique ${ }^{4}$; certaines recherche-action ne sont " plus que " des interventions ou de l'exercice de conseil; il en va de même pour la rechercheévaluation. Lorsque les objectifs ne sont pas suffisamment explicites dans le descriptif de recherche pour justifier à eux seuls la sélection ou non du projet, ces deux critères utilisés de concert se sont révélés très efficaces.

\section{Méthodologie de recherche}

La cueillette des données s'est effectuée dans un premier temps à même les sites Internet des chaires, instituts et centres de recherches et des chercheurs indépendants, en prenant pour hypothèse - une hypothèse qui ne s'est que partiellement vérifiée par la suite - que ces sites étaient à jour. Les données ont été validées par courrier électronique ou par téléphone. Par ailleurs, une série d'entrevues a été conduite auprès de représentants des sept axes de recherche privilégiés ${ }^{5}$.

Des indicateurs différents de ces sept axes sont utilisés dans d'autres travaux ${ }^{6}$, mais ils nous ont semblé moins aptes à mettre en perspective les pratiques professionnelles en communication. En dépit des problèmes que cela peut occasionner pour le développement d'analyses comparatives, nous avons jugé pertinent d'adopter les indicateurs développés dans les mandats d'ORBICOM, le Réseau mondial des chaires UNESCO en communication, et de les remodeler au besoin, en fonction des exigences de l'objet d'étude.

La classification et l'interprétation des données ont été effectuées en fonction des catégories d'analyse et des indicateurs mentionnés plus loin. Cette étape ne fut pas sans poser plusieurs problèmes majeurs. Comme le terrain - par chance pour le chercheur n'est pas aussi quadrillé qu'un échiquier, les projets se trouvent parfois être revendiqués 
par plusieurs institutions (lorsque des chercheurs de chacune y sont impliqués). D'autre part, certains chercheurs travaillent tantôt de façon indépendante, tantôt au sein d'initiatives collectives; enfin, plusieurs projets chevauchent plusieurs axes de recherche.

Nous avons donc pris en compte une nouvelle fois la perspective des chercheurs. Par exemple, si l'objet de recherche était les nouvelles technologies mais que la perspective adoptée était de regarder l'impact de ces technologies sur la communication et l'organisation du travail dans une institution bancaire, nous avons classifié le projet en fonction de cette perspective, soit dans l'axe communication organisationnelle. En ce qui concerne la détermination de l'institution principale d'accueil de la recherche dans les cas de double affiliation, nous avons retenu comme institution d'accueil celle dans laquelle le chercheur principal ou le responsable du projet exerçait.

Enfin, tous les chercheurs participant aux travaux d'un groupe ou d'un centre ont été reconnus comme affiliés à cet organisme, tandis que seuls les chercheurs n'ayant aucune affiliation pour aucun de leurs projets ont été classifiés comme chercheurs indépendants 7 . Dans le cas d'affiliations multiples le lieu de recherche impliquant le plus haut niveau d'implication ou de responsabilité a été retenu.

\section{Catégories d'analyse}

19 Un certain nombre de catégories d'analyse ont été sélectionnées comme étant les plus aptes à dégager les tendances lourdes et les tendances émergentes de la recherche en communication au Québec. En bref, il s'agit des lieux de recherche, des axes de recherche, des types de recherche, des problématiques privilégiées, des partenariats privilégiés et des questions du financement et de la diffusion de la recherche.

\section{Échantillon}

Le corpus de recherche est constitué de l'ensemble des chercheurs, des centres, des groupes, des instituts et des projets en cours au Québec ${ }^{8}$. Cela inclut 161 chercheurs actifs et 174 projets de recherche entrepris ou en cours dans la période très récente des deux dernières années, c'est-à-dire 1995-1996.

21 À moins d'être impliqués sur les projets d'autres chercheurs professionnels, les étudiants de doctorat et de postdoctorat ne sont pas compris dans le corpus de cette étude ${ }^{9}$. Pourtant les projets de thèse soutenus, subventionnés ou pas, mériteraient d'être l'objet d'un volet spécifique, dans lequel on ferait le point sur les axes, types et problématiques de recherche, les subventions accordées et l'occurrence de publications, de diffusion ou de prix ; volet que l'on pourrait comparer aux recherches professionnelles qui sont l'objet de cette étude.

\section{Limites}

Signalons quatre limites à cette recherche :

- La première est liée à la période retenue pour le corpus. Elle ne permet pas de perspective historique; nous ne pouvons que souligner les tendances lourdes et les faits porteurs d'avenir. 
- La seconde en importance est que cette recherche ne prend pas en compte la façon dont la recherche à l'étranger, en particulier en Amérique et en Europe, influence la recherche au Québec et comment, en retour, les chercheurs québécois contribuent au développement scientifique de l'étude des communications. En procédant aussi à une analyse systématique de ce qui se publie, en regardant qui publie quoi, qui cite qui et quelles perspectives théoriques prévalent pour chacun des projets, nous pourrions avoir un tableau bien différent et relever quels chercheurs, comme sources d'influence, font avancer la recherche en communication.

- Il existe encore au Québec, en 1996, des chercheurs travaillant seuls et sans subsides à d'ambitieux projets. Marginaux jusqu'au bout, ils sont peu amateurs de colloques, et ne courent pas après la visibilité. Il se peut que plusieurs d'entre eux nous aient échappé.

- Enfin, faute de distanciation critique - dans l'espace et dans le temps - la démarche est plus descriptive qu'analytique.

27 Université du Québec à Montréal, créé en 1987. Cela n'inclut pas toutes les thèses avec une importante dimension communicationnelle soutenues dans d'autres disciplines avant et après la création de ces programmes. D'où le choix de ne pas considérer les thèses de doctorat dans ce portrait de la recherche en communication au Québec.

\section{Tendances lourdes et faits porteurs d'avenir} principales institutions qui l'abritent et sous quelle forme : s'agit-il plutôt de recherches de chercheurs indépendants ou d'initiatives collectives? Quelle est la pertinence de distinguer les instituts et les chaires, des centres, des laboratoires, des réseaux et des groupes ? Y a-t-il au Québec des « appellations contrôlées » des lieux de recherche? distinguer les acteurs institutionnels majeurs de la recherche en information et communication d'après leur appellation ${ }^{10}$. À l'exception de l'appellation d'institut, qui comme en France désigne un niveau hiérarchique supérieur (dans le cadre universitaire, l'institut dépend directement du vice-rectorat à la recherche) qui fait partie d'un règlement qui qualifie l'institut d'organisme à statut particulier, et excepté aussi l'appellation de chaire, qui désigne une unité créée par le conseil d'administration de l'Université et soutenue financièrement par des organismes extérieurs, les appellations de centre, groupe, laboratoire et réseau, pour ne nommer que celles-là sont arbitraires, très variables et sans inférence directe.

31 Les universités reconnaissent un petit nombre de ces unités de recherche, lesquelles ont accès à un soutien institutionnel. Il y a là effectivement une hiérarchie par critères qui débute au statut de groupe, puis à celui de laboratoire et enfin à celui de centre de recherche. Mais pour ajouter un peu plus à la confusion, ces unités ont le libre choix des étiquettes. Ainsi, un laboratoire peut s'appeler groupe, et un groupe centre ${ }^{11}$

Dépasser cette confusion des statuts, qui nous interdit de dégager une perspective claire de l'importance de la recherche en communication au Québec en fonction du nombre et du statut des lieux de recherche, exige alors de porter une attention accrue aux programmes et aux projets eux-mêmes. 

organismes traditionnels. communications. ces deux dernières années sont :

l'université Laval (30)

l'université de Montréal (UdM) (27) polytechnique (2). en toute discrétion. compte dans le cadre de cette étude.

De l'attention portée aux lieux de recherche, une tendance se dégage clairement vers un regroupement des chercheurs dans les établissements ayant les plus importants départements de communication, en termes de nombre de personnes impliquées, et un regroupement dans les collectivités institutionnelles de recherche.

Sur les 161 chercheurs en communication que nous avons dénombrés comme étant actifs au cours des deux dernières années, 12 seulement exercent comme chercheurs indépendants à l'automne 1996. Cette concentration des lieux de recherche est sans doute une des conséquences importantes de la crise du financement de la recherche par les

Au total, on compte huit universités québécoises qui possèdent un département d'études en communication; quatre d'entre elles offrent des programmes de recherche en communication de $3 \mathrm{e}$ cycle, parmi lesquelles trois proposent un programme conjoint.

À ces départements spécialisés en communication, il faut ajouter le programme en gestion des arts - jusqu'au troisième cycle - offert par l'École des hautes études commerciales (HEC), et les activités d'enseignement et de recherche de la téléuniversité (TÉLUQ). L'essentiel de la recherche en communication est encore concentré en ces lieux. Par ailleurs, six organismes privés de recherche ont des activités dans le domaine des

Par ordre décroissant, les lieux de recherche ${ }^{12}$ au regard du nombre de chercheurs actifs

l'université du Québec à Montréal (UQAM) (25)

les institutions de recherche privées ou para-publiques (22)

la téléuniversité du Québec (TÉLUQ) (16) l'École des hautes études commerciales (HEC) (15) l'université de Sherbrooke (8) Concordia University (5)

McGill University, l'École nationale d'administration publique (4)

l'université du Québec à Chicoutimi, l'université du Québec à Trois-Rivières et l'École

Il faut signaler que seule une infime partie de la recherche privée est accessible ou officialisée. On ne pense pas ici aux organismes de recherche privés mais plutôt à toutes les commandes réalisées pour le compte de l'industrie par des organismes indépendants,

46 Nous n'avons pour l'instant aucun moyen d'évaluer l'importance de cette recherche initiée et réalisée par les entreprises privées, les associations et corporations professionnelles, ou même les ministères. C'est pourquoi elle n'a pas pu être prise en

47 Corrélativement, doit-on voir comme une tendance émergente la présence affirmée des institutions de recherche privées et, parallèlement, celle des lieux de recherche académiques dont la spécialité originelle n'est pas la communication? Présage-t-elle un déplacement des lieux de recherche en communication vers des organismes frontières entre l'université et l'industrie et allons-nous vers l'éclatement du champ disciplinaire? 


$$
\begin{aligned}
& \text { développement, les usages, l'accès et le transfert des nouvelles technologies ; un axe de } \\
& \text { recherche à la frontière de plusieurs disciplines. }
\end{aligned}
$$

Cette intuition se trouve renforcée par la prédominance des recherches portant sur le

\section{- la focalisation des axes et des problématiques de recherche autour des NTIC}

On constate en effet - voir figure ci-dessous - que la recherche autour de l'accès, de l'usage et du transfert des nouvelles technologies constitue l'axe de recherche prédominant. Il faut ajouter que la presque totalité de la recherche sur les politiques et le droit des communications et sur la communication organisationnelle et institutionnelle de même qu'une part importante de la recherche portant sur la communication internationale et le développement sont aussi directement reliées aux nouvelles technologies de l'information et de la communication.

\section{Axes de recherche}

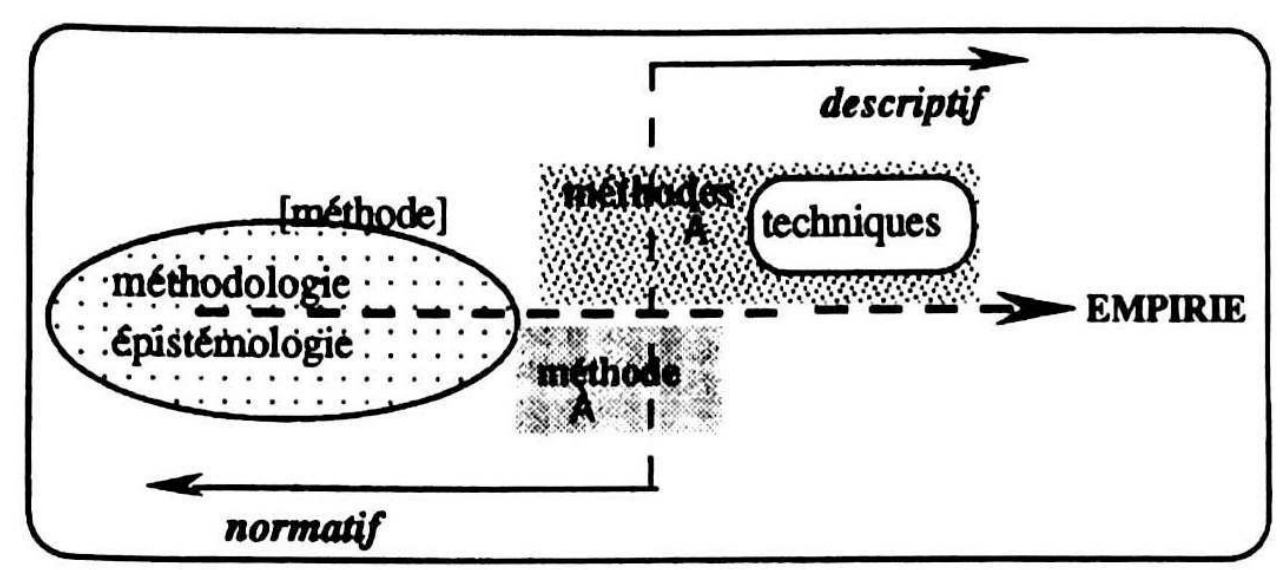

Figure 1 privilégiées par les chercheurs, on voit se renforcer cette focalisation des intérêts autour
des nouvelles technologies. Une synthèse effectuée à partir de l'ensemble des 174 projets
de recherche en cours ces deux dernières années en fait foi de manière éloquente.

PROBLÉMATIQUES PRIVILÉGIÉES PAR AXE

\section{ACCÈS, TRANSFERTS ET USAGES DES NTIC}

- Développement de l'autoroute électronique

- Dév. et expérimentation d'applications multimédias

- Interaction personne-machine et systèmes d'aide

- Usages des NTIC par des populations spécifiques

COMMUNICATION ET DÉVELOPPEMENT INTERNATIONAL

- TIC et démocratisation

- Négociation et gestion de grands projets APD

- Communications et commerce international 


\section{COMMUNICATION ORGANISATIONNELLE ET INSTITUTIONNELLE}

-Évaluation des impacts des TIC

- Implantation des TIC • Communication et changement stratégique

POLITIQUES ET DROIT DES COMMUNICATIONS

- Droit du cyberespace

- Politiques comparées de l'audiovisuel et des autoroutes de l'information

FORMATION PROFESSIONNELLE ET ÉTHIQUE

-Journalisme : - impact des femmes

- dilemmes éthiques

- crise du journalisme d'information

- Éthique et communication politique

DÉV. DES MÉDIAS ET INDUSTRIES CULTURELLES

- Réception et évaluation des produits culturels

- Télévision : - jeunes

- violence

- régions

- Histoire des industries culturelles

RELATIONS PUBL., AFFAIRES PUBL., PUBLICITÉ

- Publicité électorale, publicité gouvernementale

- Relations publiques et communication institutionnelle

Certaines problématiques semblent écartées au sein d'axes de recherche qui sont déjà relativement déshérités. Elles pourraient devenir à court terme des priorités de recherche $\mathrm{du}$ fait de leur pertinence sociale et industrielle. Par exemple, pour la pertinence sociale : la recherche sur les pratiques professionnelles en communication au regard des questions d'éthique que pose le mélange des genres journalistiques, relationnistes et publicitaires. D'autre part, la recherche sur la pratique des relations publiques est pour ainsi dire inexistante au Québec ces deux dernières années.

Pourtant on peut penser que les problèmes corporatifs reliés à la gestion et à la communication du risque et aux questions des relations publiques interinstitutionnelles et des relations publiques des institutions auprès des communautés culturelles, de même que les questions reliées à l'évaluation des relations publiques, devront tôt ou tard faire l'objet d'une attention plus soutenue de la part des chercheurs et des bailleurs de fonds institutionnels et autres, pour leur pertinence commerciale et industrielle.

Même dans le domaine pourtant surexploité de la recherche sur les NTIC, une meilleure compréhension de certains enjeux commande la recherche. La question des usages sociaux en particulier est relativement marginale; en outre, peu de chercheurs s'intéressent à la problématique pourtant cruciale de l'évaluation de ces usages.

84 Il faut bien en conclure que les problématiques porteuses, celles qui rassemblent une bonne partie des intérêts de recherche au sein de chaque axe, sont singulièrement peu nombreuses. On peut donc parler de concentration des axes et des problématiques de recherche, au détriment d'une plus grande diversité et d'une meilleure couverture de certains thèmes négligés. 
ces axes de recherches et problématiques présentent un intérêt pour plusieurs groupes de professionnels (en relations publiques et en journalisme en particulier), pour les preneurs de décisions (la recherche touchant la prise de décision, la communication et le développement international) et pour des groupes industriels (les politiques et le droit des communications).

\section{- l'impact du financement privé de la recherche sur les types de recherche et les partenariats privilégiés}

À la lumière de nos observations, on peut affirmer que les chercheurs, tout comme les responsables d'équipes de recherche, n'aiment pas aborder la question du financement, qu'il s'agisse des montants de financement qu'ils obtiennent ou de l'origine de cet argent. Plusieurs d'entre eux $(7 \%)$ refusaient toujours après plusieurs tentatives de dévoiler ces informations. La plupart se sont montrés d'emblée très hésitants, en particulier ceux qui reçoivent du financement privé. Si bien que nous n'avons systématisé que les données ayant trait à l'origine du financement, celles concernant les montants étant trop incomplètes et imprécises.

Malgré ces difficultés, nous sommes en mesure de produire une image fidèle de l'origine du financement des projets. Nous avons redistribué ce financement entre les sources en fonction de la part de chaque partenaire, dans les cas de financement mixte ${ }^{13}$.

Origine du financement de recherche

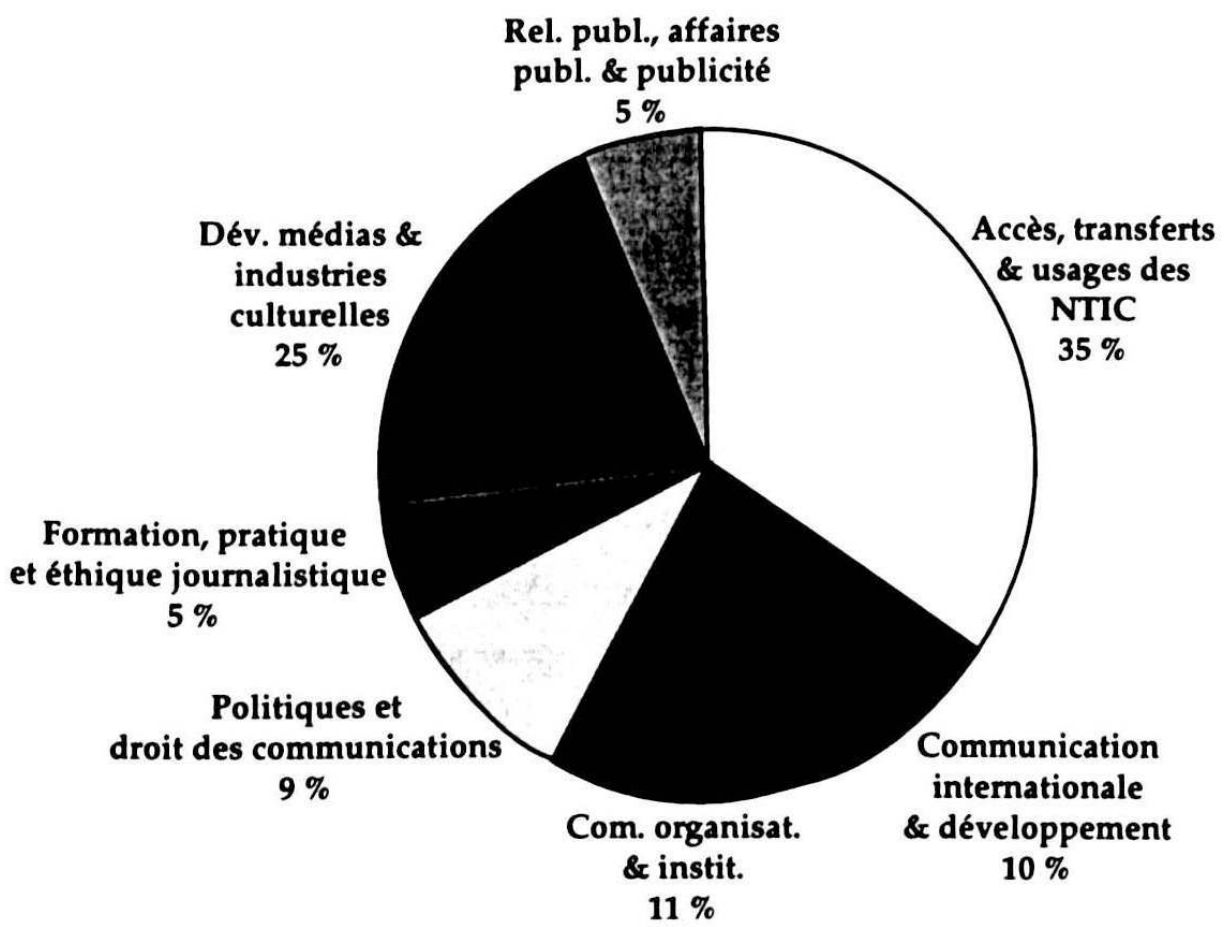

Figure 2

Parmi les partenaires corporatifs identifiés au cours de cette étude, les câblodistributeurs représentent $10 \%$ du financement total de la recherche en communication au Québec, ces 
deux dernières années. Ensemble, l'entreprise privée et les cablodistributeurs constituent la plus grosse enveloppe de financement. À cet égard, les incitatifs fiscaux jouent certainement un rôle important. En 1994 au Canada, les crédits d'impôt accordés pour la recherche et développement se chiffraient à un milliard de dollars, pour des dépenses effectuées en recherche et développement par le secteur privé atteignant un total de 6,1 milliards $^{14}$. Plusieurs des chercheurs interrogés nous ont d'ailleurs confirmé l'augmentation du nombre de leurs partenariats de recherche avec l'industrie privée, avec d'autres organismes non gouvernementaux et avec des fondations ou des organismes multilatéraux.

On peut sans doute supposer que les politiques de dégrèvement fiscal ont un impact sur les types de recherche privilégiés au Québec, incluant les recherches en communication, et qu'elles expliquent partiellement l'importance de la recherche appliquée sous toutes ses formes, puisqu'elle atteint près de $60 \%$ de la recherche effectuée ces deux dernières, en communication au Québec.

Types de recherche

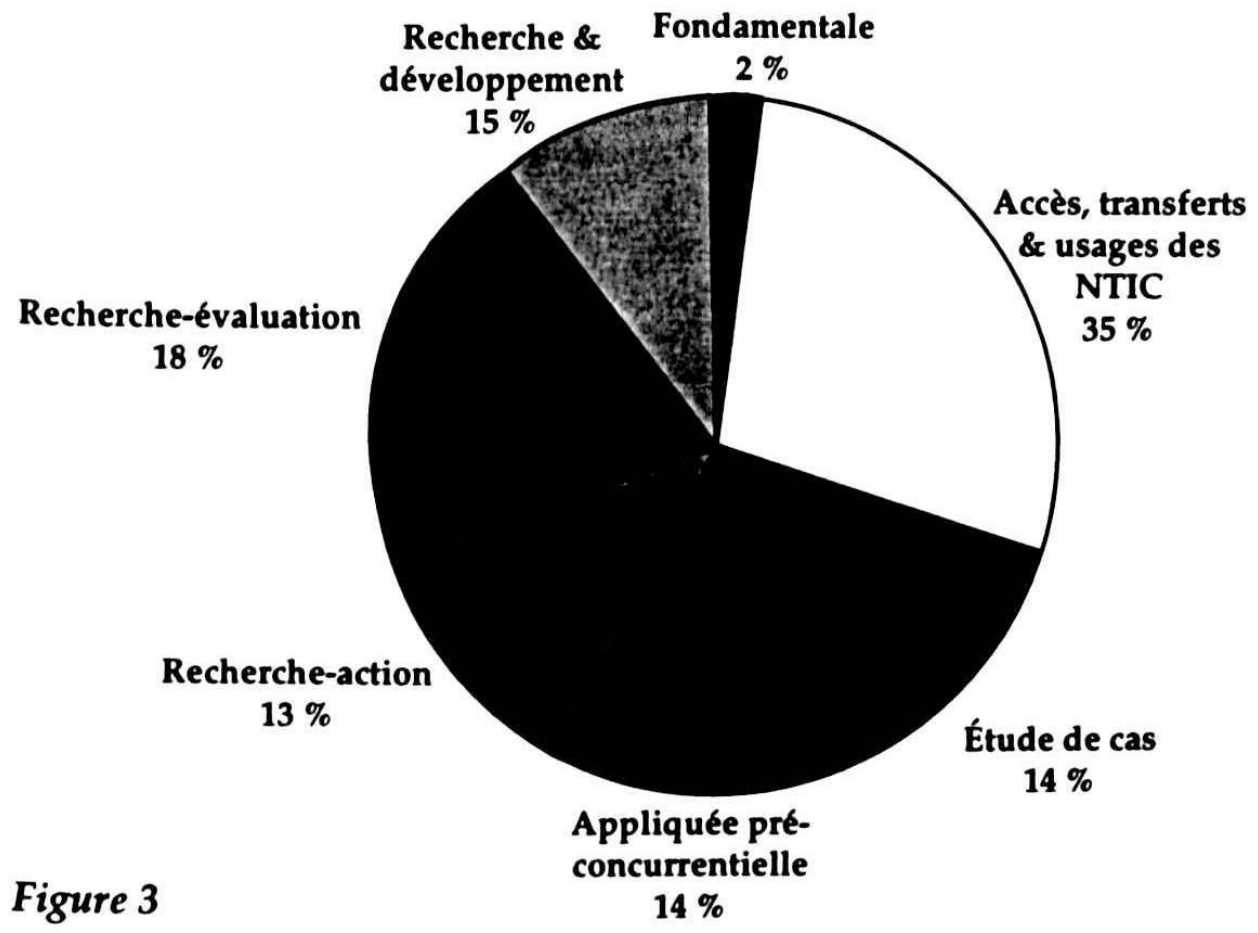

Figure 3 d'une tendance progressive de l'enracinement des types de recherche dans des 
préoccupations qui privilégient l'étude des pratiques de certains acteurs sociaux ou institutionnels spécifiques. Autrement dit, la spécificité de l'étude de cas dans le secteur de la recherche empirique en communication, au Québec, est de privilégier pragmatisme et induction, plutôt que théorie et déduction. Elle légitime la distinction que nous faisons.

4) La recherche appliquée préconcurrentielle aussi nommée pré compétitive. Il s'agit là d'une distinction récente introduite par plusieurs organismes importants de recherche en communication ${ }^{16}$. Elle désigne la recherche appliquée qui précède l'intérêt d'un seul intervenant ou acteur industriel. Le secteur de la recherche appliquée étant en développement exponentiel et les subventions de recherche gouvernementales suivant une courbe inverse, on comprend pourquoi $60 \%$ de la recherche en communication au Québec se fait déjà dans le secteur de la recherche appliquée. D est donc d'actualité et il devient urgent d'éclaircir ce concept fourre-tout et de distinguer les différentes facettes de la recherche appliquée. À cette étape d'évolution de la catégorie analytique ${ }^{17}$, on distingue la recherche appliquée préconcurrentielle de la recherche-action (tension vers l'intervention), de la recherche-évaluation (tension vers le conseil) et de la recherche et développement (tension vers la conception et la production).

5) La recherche-action (où se trouvent incluses un certain nombre d'études en communication et développement international et en communication organisationnelle).

6) La recherche-évaluation (où l'on retrouve tous les prétests, par exemple, l'évaluation des émissions télévisées pour la jeunesse, ou de la violence à la télévision).

7) La recherche et développement, habituellement oriente vers un groupe d'acteurs industriels spécifiques ou un seul client (on y retrouve en particulier la recherche sur le multimédia et les autoroutes de l'information).

La tendance lourde en faveur de la recherche appliquée peut être vue comme un impact des attitudes émergentes au niveau du financement par des organismes privés. On peut appréhender un second impact en s'intéressant aux partenariats de recherche privilégiés. En effet, une importante proportion de la recherche s'effectue dans le cadre de collaborations multisectorielles, internationales ou multidisciplinaires ou encore de collaborations multiparticipatives, c'est-à-dire combinant tous ces partenariats. 


\section{Partenariats de recherche}

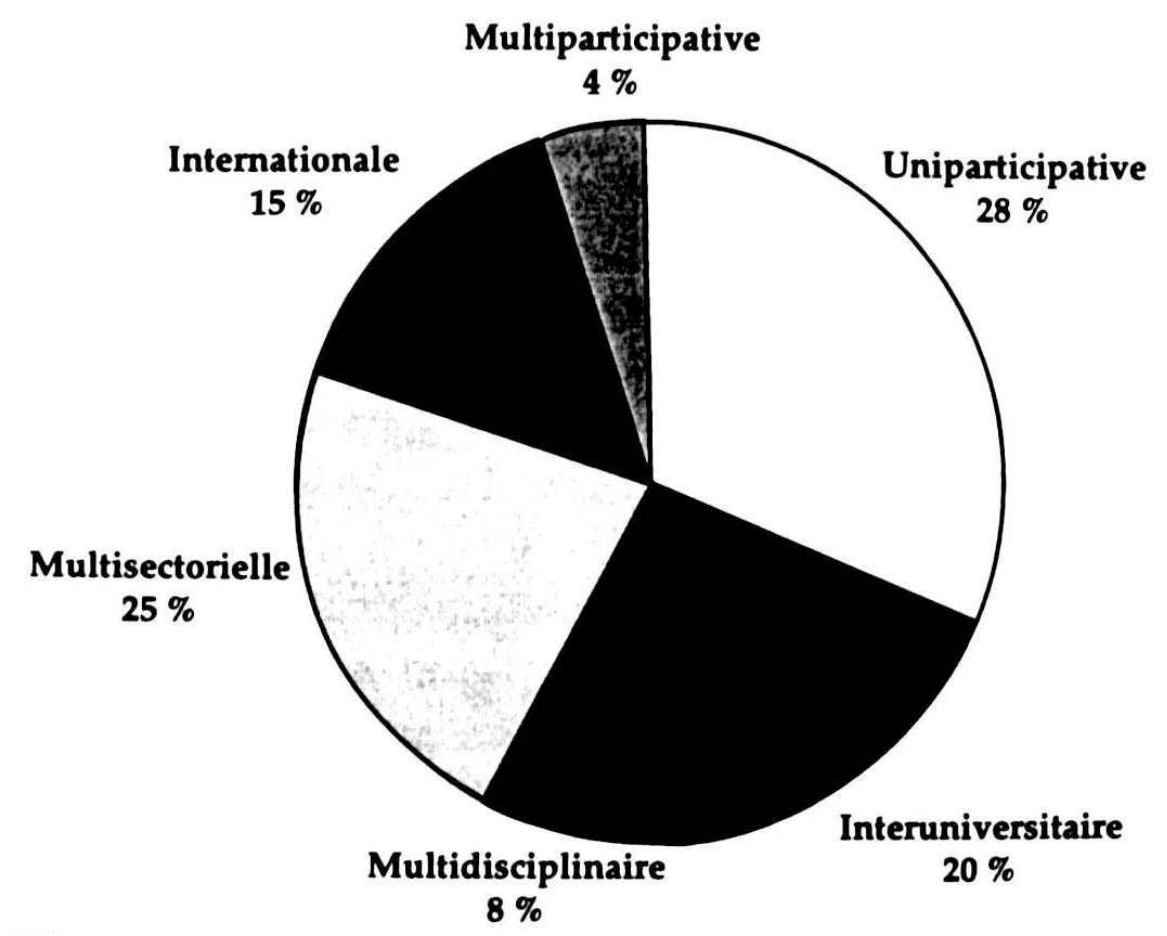

Figure 4

Nous distinguons en effet les partenariats uniparticipatifs, c'est-à-dire ceux qui impliquent des chercheurs d'une seule discipline, d'un seul pays, d'un seul secteur (le secteur universitaire en général), des partenariats qui regroupent des chercheurs de plusieurs disciplines (par exemple communication, relations industrielles et droit), de plusieurs pays, ou dans plusieurs secteurs (par exemple universitaire et corporatif ou universitaire et gouvernemental) ${ }^{18}$.

100 La diminution du financement traditionnel à la recherche, dorénavant pris en main par de nouveaux partenaires industriels et professionnels, implique une concentration des chercheurs au sein de plus grandes équipes de recherche; équipes investies sur d'importants projets, impliquant d'autres chercheurs dans d'autres disciplines, d'autres régions du monde et impliquant surtout de nombreux partenariats $(29 \%)^{19}$ avec des professionnels ou des experts de l'industrie privée.

101 La plupart de ces projets sont en œuvre sur l'ensemble de la décennie. La recherche se concentre donc à plusieurs titres mais, parallèlement, les programmes de recherche en partenariat s'étendent sur de longues périodes.

Ces partenariats multiples ont aussi une influence sur la diffusion de la recherche en communication réalisée au Québec. On observe qu'elle emprunte surtout les voies traditionnelles que sont la publication dans des revues scientifiques et la participation à des colloques et congrès internationaux. 


\section{- la visibilité des chercheurs québécois au Québec, au Canada et à l'étranger}

Doit-on s'inquiéter du peu d'ouvrages monographiques ${ }^{20}$ publiés par les chercheurs québécois en communication? Leur présence publique via les médias à grande diffusion est marginale, quel que soit le média: articles dans la presse, émissions radio ou télédiffusées ou encore diffusion de la recherche sur Internet.

Comment les chercheurs prennent-ils leur place dans la société civile du Québec ? À certaines occasions, des chercheurs québécois en communication ont acquis une importante notoriété, lors de leur participation à des commissions d'enquêtes sur les industries culturelles. Ces événements ont donné lieu à des rapports de recherche ayant largement circulé dans tous les milieux, y compris à l'étranger, et ayant fait l'objet d'une forte diffusion médiatique donnant lieu à un véritable débat de sociétée ${ }^{21}$. Ce n'est pas le cas dans la période récente des deux dernières années.

\section{Diffusion de la recherche}

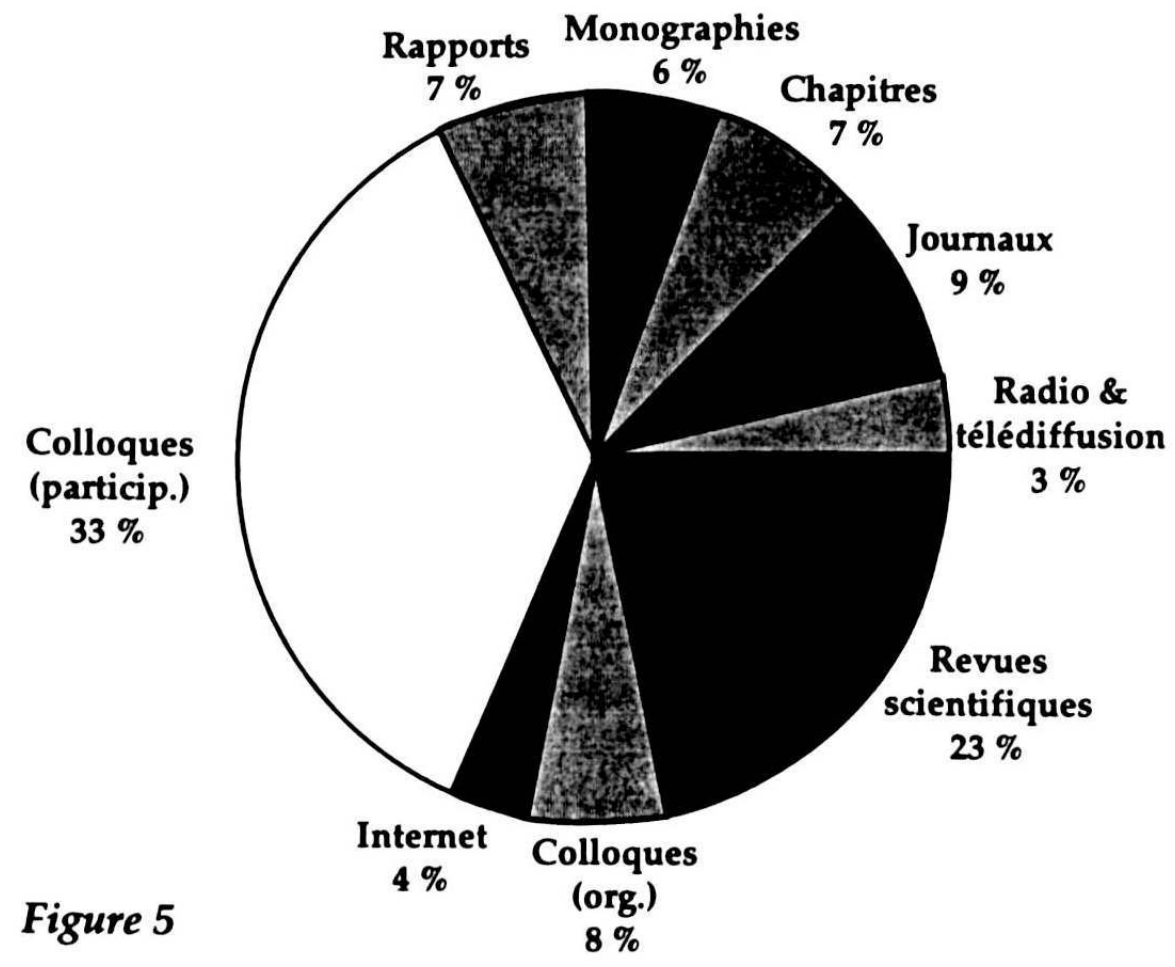

Figure 5

Qui plus est, nous avons observé qu'une part non négligeable de la recherche ne donne lieu à aucune diffusion ou presque : à l'occasion un article, un rapport de recherche, une participation dans un colloque local, tout au plus. Voilà qui ne joue pas en faveur de la visibilité des chercheurs québécois en communication, ni non plus en faveur de l'émergence d'une culture disciplinaire forte, moins éclatée, qui pourrait conduire à une synergie de l'ensemble des courants de recherche en communication au Québec. 


\section{Conclusion}

106

De cette photographie prise sur le vif, nous pouvons dégager non seulement les traits saillants de la recherche en communication réalisée au Québec ces deux dernières années, mais peut-être aussi quelques flottements, que nous pourrions interpréter comme des glissements vers de nouvelles tendances en émergence.

Plusieurs faits porteurs d'avenir se dessinent et paraissent converger: la recherche semble vouloir - ou devoir - combiner idées conceptuelles et terrains pragmatiques, au profit de ces derniers; les chercheurs se regroupent au sein d'équipes multidisciplinaires et internationales et se concentrent sur un petit nombre de problématiques, généralement reliées au NTIC; et surtout, la crise du financement traditionnel semble jouer comme l'élément déclencheur du remodelage des axes, des types, des problématiques et des partenariats de recherche en communication au Québec.

8 À l'origine du projet, nous voulions situer la place qu'occupe la recherche sur les pratiques professionnelles dans l'ensemble de la recherche québécoise en communication, afin d'évaluer l'impact de cette recherche sur les autres courants de recherche et sur les pratiques professionnelles.

Force est de constater que nous n'avons fait que lever le voile sur une première approche de la recherche en communication au Québec, axée sur les pratiques professionnelles. On n'en demeure pas moins surpris du peu d'intérêt manifesté ces deux dernières années pour les études sur la transformation des pratiques professionnelles en journalisme, en relations publiques et en publicité, mais également pour la recherche fondamentale, en particulier pour les analyses critiques et les études de cas.

10 Il ressort aussi de cette étude qu'il serait urgent de réfléchir au rôle des chercheurs dans la sélection et la formulation des problématiques de recherche, principalement à une période où les partenariats multiples augmentent. À cet égard, on peut s'interroger aussi sur l'accès aux résultats des recherches, multisectorielles en particulier, ainsi que sur les usages que l'on fait de ces résultats.

111 Aussi, ne devrait-on pas s'intéresser à la transformation des pratiques, au regard de ces résultats de recherche et, parallèlement, à la transformation de l'emploi au regard de la formation en recherche. Comme on pourrait se poser la question de savoir si les sciences humaines, et en particulier les sciences de la communication, bénéficient, autant que d'autres disciplines scientifiques, des investissements privés déjà protégés par les déductions fiscales sous la forme des crédits d'impôts.

Toutefois, il y a sans doute déjà une leçon à tirer de cette photographie sur le vif: elle nous invite à favoriser le développement de la recherche sur les pratiques professionnelles en communication, leur permettant de jouer le rôle important qu'elles sont appelées à avoir dans le milieu universitaire et au sein des secteurs professionnels concernés 


\section{NOTES}

1. Par axe de recherche nous entendons un domaine de recherche distinct. Cette étude en distingue sept. Voir note infrapaginale $n^{\circ} 6$.

2. . Il est entendu que la quasi-totalité de la recherche conduite par les industries des médias, les cabinets-conseils en relations publiques et les agences de publicité nous échappe, faute d'accès à l'information

3. . Ici la perspective est comprise comme le cadrage du chercheur, son optique sur l'objet spécifique qu'il vise à connaître

4. . Ex. : développement d'algorithmes

5. Ce sont: l'accès, le transfert et les usages des NTIC; la communication internationale et le développement (incluant les communications interculturelles, la prise de décision multilatérale et la recherche sur les multinationales des communications) ;la communication organisationnelle et institutionnelle; les politiques et le droit des communications ; la formation professionnelle et l'éthique (on y retrouve la recherche sur la pratique journalistique, en particulier); le développement des médias et les industries culturelles (incluant les recherches comparatives avec des médias étrangers, la recherche sur l'édition, sur la muséologie.) les relations publiques, les affaires publiques et la publicité.

6. Ce sont rarement les mêmes d'une étude à l'autre, d'ailleurs

7. Excepté que dans la liste des affiliations présentée ci-aprés sous le titre « la concentration des lieux de recherche", les chercheurs ont été réintégrés dans leur organisme d'appartenance premier, afin de ne pas créer de distorsions qui pourraient être mal interprétées. Par exemple qu'il soit considéré qu'il n'y ait aucun chercheur en communication actif ces deux dernières années à l'université du Québec a Trois-Rivières, sous prétexte que tout deux aient été comptés dans un groupe appartenant a une autre institution

8. . Cette étude est exhaustive. Toutefois, environ $3 \%$ de l'information n'a pu être obtenue faute de collaboration. Dans la plupart de ces cas, nous disposons tout de même de certaines données. Par ailleurs, il est toujours possible que des chercheurs ou des groupes marginaux aient pu nous échapper

9. . Depuis la création du programme d'études graduées en communication de McGill University en 1975, 44 étudiants y ont soutenu leur thèse de doctorat, tandis que 31 étudiants ont soutenu leur thèse de doctorat dans le cadre du programme conjoint de doctorat en communication Concordia University-Université de Montréal

10. C'est pourquoi nous avons renoncé à présenter ici un tableau des centres, chaires et groupes par institution, même si nous disposons de ces informations.

11. Nous mentionnerons tout de même avoir dénombré trois instituts de recherche, trois chaires et 34 groupes, laboratoires, centres, réseaux, etc. Il arrive qu'une chaire soit elle-même rattachée a un centre (ex: la chaire en relations ethniques de l'université de Montréal est rattachée au Centre d'études ethniques de cette même université) et cela vaut aussi pour plusieurs groupes de recherche.

12. . Ces lieux de recherche n'incluent pas seulement les chercheurs rattachés aux départements en communication. Par exemple, les chercheurs du département d'information et de communication de l'université Laval ne constituent qu'une partie des chercheurs de cette université qui travaillent sur des projets de recherche en communication 
13. . Le Fonds pour la formation de chercheurs et l'aide à la recherche (FCAR) est un organisme québécois, tandis que le Conseil canadien de recherche en sciences humaines du Canada (CRSH) est un organisme canadien.

14. . Source: Centre interuniversitaire de recherche en analyse des organisations (CIRANO) "Analyse de l'effet des incitations fiscales sur les dépenses des entreprises en recherche et développement", abstract de projet de recherche dans Programmation de recherche détaillée 1996-1997. (Résolution du conseil d'administration du 8 mai 1996) sur Internet: cirano@cirano.umontreal.ca

15. . Les types de recherche peuvent être distingués en fonction de l'objectif qui est poursuivi : veut-on expliquer, comparer, analyser, évaluer, changer ou concevoir?

16. . Centre de recherche pour le développement international (CRDI), Centre francophone de recherche en informatisation des organisations (CEFRIO), Centre de recherche informatique de Montréal (CRIM) ; rapports annuels 1995-1996

17. . Il est entendu que nous adoptons la position du chercheur qui préfère adapter ses outils au terrain qu'il explore plutôt que d'avoir à faire l'inverse. Ces catégories analytiques sont donc à la fois issues du vocabulaire développé par les chercheurs eux-mêmes pour désigner leur pratique et de la démarche progressive sur ce terrain de recherche. Ainsi nous espérons avoir produit une image plus juste des types de recherche en communication tout en vérifiant l'étanchéité de ces catégories lors du processus de classification.

18. . Il est difficile d'éliminer complètement le recoupement d'une catégorie à l'autre. Par exemple, un programme de recherche ou un projet de recherche dans le cadre d'un partenariat international peut aussi être un partenariat de recherche multidisciplinaire. C'est pourquoi, comme dans le cas du financement, nous avons redistribué le projet en fonction des genres de partenariats différents qu'il impliquait, a moins qu'il ne recoupe toutes les autres formes de partenariats, auquel cas il entre dans la catégorie recherche multiparticipative À noter que la catégorie recherche internationale désigne les projets conjoints en communication, d'équipes de chercheurs de différents pays, impliquant des chercheurs québécois, et qu'elle ne désigne pas la nature de la problématique analysée

19. $25 \%$ des 174 projets de recherche en communication au Québec constituant des partenariats multipariels, plus le $4 \%$ de recherche multiparticipative, laquelle inclut nécessairement des projets multisectoriels.

20. . Auteurs uniques ou " readers ».

21. . Le rapport d'enquête de la commission Sauvageau-Caplan, par exemple.

\section{AUTEURS}

\section{ANNE-TAMARA LORRE}

Chercheure

\section{THÉRÈSE PAQUET-SÉVIGNY}

Titulaire chaire UNESCO-BELL en communication et développement international 